\title{
When is chemotherapy in head and neck squamous cell carcinoma not indicated?
}

\author{
Missak Haigentz Jr. • Jan B. Vermorken • Arlene A. Forastiere • June Corry • Jonathan J. Beitler • \\ Primož Strojan • Dana M. Hartl • Juan P. Rodrigo $\cdot$ Carol R. Bradford • Alessandra Rinaldo • \\ Robert P. Takes $\cdot$ William M. Mendenhall $\cdot$ Ashok R. Shaha $\cdot$ Gregory T. Wolf $\cdot$ Alfio Ferlito
}

Received: 30 December 2013 / Accepted: 16 January 2014 / Published online: 14 February 2014

(C) Springer-Verlag Berlin Heidelberg 2014

\section{Introduction}

Given the widespread availability of several effective multidisciplinary care plans, the decision-making and clinical management for the patient with head and neck squamous cell carcinoma (HNSCC) are complicated. Although national guidelines like the National Comprehensive Cancer Network (NCCN) guidelines in the United States exist for patient care by disease site and stage of disease [1], the choice of therapeutic approach for a particular patient should be based on goals of care, patient functional status and comorbidities, and morbidities

This paper was written by members of the International Head and Neck Scientific Group (www.IHNSG.com).

M. Haigentz Jr.

Division of Oncology, Department of Medicine, Albert Einstein College of Medicine, Montefiore Medical Center, Bronx,

NY, USA

J. B. Vermorken

Department of Medical Oncology, Antwerp University Hospital, Edegem, Belgium

\section{A. A. Forastiere}

Department of Oncology, The Sidney Kimmel Comprehensive Cancer Center, Johns Hopkins University School of Medicine, Baltimore, MD, USA

\section{J. Corry}

Division of Radiation Oncology, Peter MacCallum Cancer

Centre, Melbourne, VIC, Australia

\section{J. J. Beitler}

Departments of Radiation Oncology, Otolaryngology,

Hematology and Medical Oncology, Georgia Research Alliance

Cancer Scientist, Winship Cancer Institute, Emory University,

Atlanta, GA, USA associated with treatment. The goals of HNSCC therapy can be categorized as either curative, employing either surgery and/or radiotherapy as a therapeutic backbone, or palliative, where symptom management and maintenance or improvement of quantity and quality of life are the primary focus.

Systemic therapies (chemotherapy and targeted molecular therapeutics) have been an important addition to the therapeutic armamentarium against HNSCC. Although generally palliative when employed as a single treatment modality, systemic therapy concurrent with radiation has become an important curative option for patients with advanced locoregional disease as initial therapy or in the high-risk postoperative setting. Randomized clinical trials have demonstrated that systemic therapy concurrent with

\section{P. Strojan}

Department of Radiation Oncology, Institute of Oncology,

Ljubljana, Slovenia

D. M. Hartl

Department of Otolaryngology-Head and Neck Surgery, Institut Gustave Roussy, Villejuif Cedex, France

\section{M. Hartl}

Laboratoire de Phonétique et de Phonologie, Sorbonne Nouvelle, Paris, France

\section{J. P. Rodrigo}

Department of Otolaryngology, Hospital Universitario Central de Asturias, Oviedo, Spain

\section{J. P. Rodrigo}

Instituto Universitario de Oncología del Principado de Asturias,

Oviedo, Spain

C. R. Bradford · G. T. Wolf

Department of Otolaryngology-Head and Neck Surgery,

University of Michigan, Ann Arbor, MI, USA 
radiotherapy improves clinical outcomes-organ preservation rates, locoregional disease control, and survival-as compared to radiotherapy alone. However, given the potential for enhanced toxicity, overtreatment, and inefficient use of healthcare resources, the selection of patients who can benefit from systemic therapy is critical. In this editorial, we briefly review the nonstandard uses of this important treatment modality and identify patient populations for whom such therapy may be challenging, with the goals of guiding high-quality, individualized, and evidence-based treatment plans for HNSCC patients and of highlighting specific areas requiring attention for the design of future clinical trials.

\section{Nonstandard/investigational applications of systemic therapy}

In the following clinical situations, there is inadequate data to support clinical benefit from the use of systemic therapies, and their application is therefore investigational.

\section{Early/intermediate stage disease}

Systemic therapy should not be administered concurrently with radiotherapy for early or intermediate stage HNSCC

Concurrent chemoradiotherapy (or in the case of use of cetuximab, bioradiotherapy) is a recommended treatment option for patients with locoregionally advanced (stages III and IV) HNSCC. With the exception of stage II nasopharyngeal cancers for which chemoradiotherapy is currently considered standard [1], the use of chemoradiotherapy or bioradiotherapy for earlier stages of disease (i.e., stages I and II, lymph node negative) is not recommended outside of clinical trials due to the generally

\footnotetext{
A. Rinaldo $\cdot$ A. Ferlito $(\square)$

ENT Clinic, University of Udine, Piazzale S. Maria della

Misericordia, 33100 Udine, Italy

e-mail: a.ferlito@uniud.it

R. P. Takes

Department of Otolaryngology-Head and Neck Surgery, Radboud

University Medical Center, Nijmegen, The Netherlands
}

W. M. Mendenhall

Department of Radiation Oncology, University of Florida, College of Medicine, Gainesville, FL, USA

A. R. Shaha

Department of Surgery, Memorial Sloan-Kettering Cancer

Center, New York, NY, USA excellent outcomes with either surgery or radiotherapy alone.

Altered radiotherapy fractionation regimens

Systemic therapy should not be administered concurrently with altered radiotherapy fractionation regimens

Despite known survival benefits of treating locoregionally advanced HNSCC with either concurrent chemoradiotherapy or altered fractionated radiotherapy regimens over standard radiotherapy fractionation alone [2-4], efforts to further intensify therapy by the addition of chemotherapy to accelerated radiotherapy plans have failed to improve on clinical outcomes over conventional chemoradiotherapy in recently published randomized phase III trials (RTOG 0129 and GORTEC 99-02) [5, 6]. With hopes to further improve outcomes, the Radiation Therapy Oncology Group (RTOG) is currently using mildly accelerated radiation (6 fractions per week as in the DAHANCA $6 \& 7$ trial [7] and the IAEA-ACC trial [8]) with concurrent chemotherapy as the standard arm in its trials. However, outside of a clinical trial, chemotherapy should be administered concurrently with conventionally fractionated radiotherapy [9].

Adjuvant or maintenance therapy

Systemic therapy should not be used as an adjuvant or maintenance therapy for non-nasopharyngeal HNSCC

The study of cytotoxic agents following radiotherapy or chemoradiotherapy for HNSCC has been hampered due to poor patient tolerance. The use of novel biological agents that can be administered orally or enterally may have greater promise in this regard, and several clinical trials of this approach are ongoing or in development. For nasopharyngeal cancer, adjuvant chemotherapy may not be essential for improving overall survival [10], but studies of Epstein-Barr viral load offer a rational approach for selective treatment of high-risk patients.

Chemotherapy alone with curative intent

Do not use systemic therapy as a single modality for HNSCC when the treatment goal is cure

A few recent studies of chemotherapy as a single treatment modality have demonstrated proof of concept of long-term patient survival and disease control in very highly selected patients with laryngeal cancer [11-13]. However, this highly novel approach remains experimental and should not be performed outside of a clinical trial $[14,15]$. 
Intra-arterial (IA) chemotherapy

IA chemotherapy, despite demonstrated feasibility and efficacy in multi-institutional settings, is not superior to intravenous chemoradiotherapy and remains experimental

IA chemotherapy has been evaluated for over 50 years to exploit pharmacokinetics of a number of cytotoxics. More recently studied, high-dose IA cisplatin can be administered locally, while systemic toxicities are reduced by a simultaneous intravenous infusion of sodium thiosulfate [16]. The bulk of the literature shows that IA administration via a femoral catheter is feasible for squamous cell carcinoma of the oral cavity, oropharynx, larynx, and hypopharynx with acceptable systemic toxicity. Toxicities related to IA therapy include tumor necrosis, bleeding, and, more rarely, cerebrovascular events [17]. Similar to intravenous drug administration, the response to neoadjuvant IA cisplatin may also be predictive of final oncologic outcomes after definitive surgery or radiation therapy [18]. Feasibility and effectiveness of concomitant IA cisplatin and radiation therapy (RADPLAT) were shown in a prospective multi-institutional study [16], with complete responses in greater than $80 \%$ of patients. High-dose IA cisplatin with concomitant hyperfractionated radiation therapy has also been shown to be feasible with complete response rates of greater than $85 \%$ for advanced and unresectable tumors $[19,20]$.

Despite these initial promising results, the only published randomized phase III trial of IA cisplatin versus concomitant intravenous chemoradiation in patients with locoregionally advanced, (functionally) unresectable HNSCC failed to demonstrate improved oncologic outcomes with IA therapy [21]. The extent of toxicities between the two treatment arms was comparable, though the sites of toxicity differed; notably, serious (grade 3 or 4) nephrotoxicity was more common in the intravenous arm while neurotoxicity was more prevalent in the IA arm. Moreover, recently published 5-year quality of life evaluations from this trial did not detect significant differences between the two approaches [22]. As a result, this mode of therapy remains investigational and should not be performed outside of a clinical trial.

\section{Re-irradiation for recurrent or second primary HNSCC}

Particular caution must be exercised when systemic therapy is used in combination with re-irradiation for selected patients

The use of re-irradiation following failure of initial therapy of HNSCC has some limited efficacy. In 2008, the Groupe
d'Etude des Tumeurs de la Tête Et du Cou (GETTEC) and Groupe d'Oncologie Radiothérapie Tête Et Cou (GORTEC) reported on the results of a small $(N=130$ patients) phase III randomized trial of postoperative re-irradiation combined with chemotherapy compared with salvage surgery alone [23]. Although locoregional control and disease-free survival were improved in the combined chemotherapy and re-irradiation arm, no improvement in overall survival was noted, and the combined regimen was associated with increased severe toxicities and several treatment-related deaths.

In the setting of unresectable locoregionally recurrent or second primary cancers, in view of the morbidities associated with and limited efficacy of re-irradiation, the role of concomitant cytotoxic chemotherapy or biologic therapy is unclear because of lack of randomized data. When systemic therapy is considered, particularly when patients have been previously treated with chemoradiation which appears to be an adverse prognostic factor for survival [24], combining re-irradiation with cetuximab deserves study. Currently, there is no standard approach for combining chemotherapy with re-irradiation [25].

\section{Special populations}

Systemic therapy is rarely devoid of toxicity, and caution must be exercised in its use. That said, few absolute contraindications exist for the use of systemic cancer therapies, and those that exist are usually specific to the toxicity profiles of the chemotherapeutic agents employed.

Although a full discussion of the toxicities of chemotherapeutic agents is beyond the scope of this editorial, there are several specific clinical scenarios where alterations in therapy or omission of chemotherapy should be considered in the management of HNSCC. It should certainly be appreciated that elderly patients, those with organ dysfunction or poor performance status (PS), as well as those with human immunodeficiency virus (HIV) infection have generally been under-represented from clinical trials; these exclusions weaken our recommendations.

\section{Elderly patients}

The recognition of cancer as a disease of aging has led to increased focus on clinical outcomes and tolerability of cancer therapies in patients with advanced age. However, clinical research involving this commonly encountered patient population is limited by few prospective studies and widely variable definitions of "the elderly."

Advanced age at HNSCC diagnosis is not an absolute contraindication for systemic therapies; however, it is one of many variables-including organ function, comorbid 
burden, polypharmacy, nutritional status, cognitive function, socioeconomic issues, geriatric syndromes, and patient preferences-that must be considered in patient management [26]. Although the MACH-NC showed a modest but significant survival benefit with the addition of chemotherapy to radiotherapy for HNSCC, it did not confirm this benefit in the subset of patients over 70 years of age [2], highlighting the need for prospective trial data for this population. Cetuximab has often been considered for elderly patients given its generally tolerable toxicity profile and lack of drug-drug interactions; however, survival benefit in patients 65 years and older and those with poor functional status was not demonstrated in subset analyses of the definitive trial led by Bonner et al. [27].

Patients with organ dysfunction

The use of systemic therapy in patients with organ dysfunction is a frequently encountered clinical challenge. Chemotherapy toxicities may result in exacerbating pre-existing renal dysfunction or hearing loss (e.g., with cisplatin), neuropathy (e.g., with paclitaxel), or bone marrow dysfunction (all cytotoxic agents), and impaired metabolism or clearance of the drug can certainly enhance toxicities. Baseline renal dysfunction may lead to increased toxicity of methotrexate, and hepatic dysfunction increases the toxicity of taxanes. Although cetuximab has been advocated by some for use in patients with pre-existing renal insufficiency or other organ dysfunction, it is not known whether clinical outcomes or toxicities are similar in otherwise fit patients.

Patients with poor functional (performance) status

Careful consideration of regimen tolerability must be weighed in the care of HNSCC patients with impaired functional status. Although disease-associated symptoms can greatly improve with induction chemotherapy approaches for previously untreated disease and serve as a bridge to subsequent chemoradiotherapy [28], induction chemotherapy has not improved survival [29-31] and should only be administered in tertiary centers [1]. Moreover, patients with impaired functional status were not included in the prospective studies of induction chemotherapy, and the acute toxicities of this approach and the potential for additional treatment-associated mortality must be recognized [28].

There have been rare studies that have investigated therapies focused on patients with poor functional status, known to be associated with poor outcomes in patients with incurable HNSCC [32]. The Eastern Cooperative Oncology Group (ECOG) recently reported results of a randomized, placebo-controlled, phase III clinical trial of weekly docetaxel with or without gefitinib for patients with unresectable recurrent and/or metastatic HNSCC specifically with an ECOG PS of 2 (ECOG 1302) [33]. The study accrued 270 patients before closure at interim analysis. The median overall survival of patients treated with docetaxel was only 6.0 months, which was not improved with the addition of gefitinib (median overall survival $=7.3$ months; hazard ratio 0.93 ; $95 \%$ confidence interval 0.72 to 1.21 ; $P=0.60$ ). Although single agent palliative therapy may be considered for PS 2 patients, patients with worse functional status at clinical presentation (ECOG PS $\geq 3$, i.e., bedbound more than $50 \%$ of the day) are often best served with supportive and palliative care.

Patients infected with HIV

In the era of highly active antiretroviral therapies (HAART), the life expectancy of persons with HIV infection has dramatically improved. Although sharp declines have been observed in incidence of malignancies associated with severe immunosuppression (i.e., Kaposi sarcoma, non-Hodgkin's lymphoma, and cervical carcinomatermed AIDS-defining cancers), the prevalence of other malignancies (i.e., the non-AIDS-defining cancers) has concomitantly increased due to aging [34].

Several epidemiological studies have additionally noted a threefold excess of HNSCC prevalence in HIV-positive populations $[34,35]$. Although the known association with oncogenic human papillomavirus (HPV) with oropharyngeal HNSCC has led some to speculate viral co-infection as an explanation for the increased risk of HNSCC in HIV-positive populations, preliminary data from HNSCC specimens of these patients have not clearly supported this hypothesis [36]. Concerns of using systemic therapies for this population include risks of immunosuppression and increased toxicity; however, limited evidence suggests that appropriately selected patients can tolerate organ-preserving therapy [37, 38]. Evidence-based guidelines are lacking to guide appropriate management of this patient population frequently excluded from participation in cancer clinical trials $[39,40]$.

\section{Discussion}

There is no doubt that chemotherapy and biological therapies have important roles in the management of patients with HNSCC-including radiosensitization as well as systemic effects. The MACH-NC demonstrated a $6.5 \%$ absolute survival benefit associated with the concurrent addition of chemotherapy with radiotherapy regimens [2], which though significant, leaves much room for improvement. Thus, in the absence of major improvements in survival, therapy-associated toxicities and patient-related factors as well as known risk-benefit ratios must be carefully 
considered in selecting optimal treatment approaches. Understanding the varied toxicities associated with combined therapy provides even more reason to have such patients treated by teams that have experience with such treatments, including an experienced medical oncologist.

Cetuximab, with known systemic and radiosensitizing effects, is the only approved biological agent for HNSCC patients and has a markedly different toxicity profile compared to cytotoxic agents. Although the toxicities of cetuximab are generally manageable, severe toxicities have been noted with the agent (e.g., hypomagnesemia, rare hypersensitivity reactions, and others including interstitial pneumonitis and cardiovascular toxicities). Given the negative results from the RTOG 0522 trial, cetuximab should not be combined with cisplatin-based chemoradiation regimens [41], and its use in combination with other chemoradiotherapy regimens must be in the context of clinical trials.

Given its generally favorable toxicity profile, cetuximab has often been advocated as an alternative for patients who may not otherwise be candidates for chemotherapy. However, subset analyses of the study resulting in its approval indication have not demonstrated survival benefit in patients 65 years of age and older and in patients with impaired PS [27], and controlled studies about tolerance of bioradiotherapy in non-trial patients that are frequently seen in daily practice are lacking. The same is true for the recurrent/metastatic disease setting, where most data on the tolerance of cetuximab in combination with chemotherapy were obtained in patients with ECOG PS of 0 and 1 [42]. However, as a single agent, tolerance is generally acceptable, and most trials using the agent in HNSCC patients with platinum-refractory disease with a WHO PS of $60 \%$ or more (thus including patients with ECOG PS 2), have shown minimal side effects [43]. Persons with incurable malignancy and very poor functional status (ECOG PS 3 or worse) may best be served with supportive and palliative care, since systemic therapy in this setting has unproven benefit.

It is important to consider that radiotherapy alone is effective and a curative-intent treatment modality for HNSCC offering organ preservation, or, in the case of previously resected disease, improved locoregional control and survival outcomes. Thus, most patients with locoregionally advanced disease who either decline concurrent systemic therapy or are deemed to be poor candidates for it can still receive and benefit from radiotherapy. The effectiveness of radiotherapy can be further improved with altered fractionation regimens [3, 4], particularly for patients with good functional status.

It is also important to note that the above considerations are all clinical in nature, with risk-benefit analyses obtained via individual clinical trials and published meta-analyses; for optimal judgment on how intensive a patient's treatment regimen should be, identification of novel prognostic and predictive biomarkers will be of critical importance.

The identification of oncogenic HPV infection in a subset of oropharyngeal cancers associated with improved patient prognosis has resulted in several prospective investigations testing altered deintensification therapy plans based on this favorable biomarker. As previously untreated HPV-associated disease is remarkably chemosensitive and radiosensitive [44], the respective roles of chemotherapy and radiotherapy as well as the use of novel surgical options [45] in treatment deintensification need to be further explored in carefully designed prospective trials. In contrast, HPV-negative HNSCC is associated with remarkably poor prognosis despite current therapies, and continued therapeutic intensification efforts under investigation will need to be tempered by patient tolerance.

\section{Conclusions}

The care for patients with HNSCC has always been a trade-off between maximizing curative potential while minimizing therapy-related morbidity, and the stepwise advances in multidisciplinary care resulting in current standard therapeutic approaches that have resulted from decades of clinical research. Advances in supportive care, chemotherapy, biological therapy and in radiotherapy derived from this research have permitted increasingly challenging treatment regimens with goals of treatment intensification, to the point where deintensification can today be considered for patients with HPV-associated disease. However, the negative trial data from several recent randomized studies must provide important pause against accepting novel intensification (or deintensification) approaches without rigorous evaluation $[9,28]$. The clinical situations discussed in this editorial whereby the use of systemic therapy is not recommended highlight areas for further investigation.

Given the paucity of data available for common populations encountered in practice, comparative effectiveness research and prospectively conducted clinical trials are urgently needed for HNSCC in the elderly and in patients with poor organ function. Patients with HIV infection are also under-represented in HNSCC trials and this population warrants prospective research, either in specific trials or as a formal stratification variable in existing randomized studies. Despite the known advances in the management of HNSCC afforded by systemic therapies, a number of important clinical questions remain for specific clinical settings and patient populations; these questions can only be addressed by supporting and accruing patients to well-designed existing and future clinical trials for this disease. 


\section{References}

1. National Comprehensive Cancer Network (NCCN) Guidelines, Head and Neck Cancers, Version 2.2013

2. Pignon JP, le Maître A, Maillard E, Bourhis J, MACH-NC Collaborative Group (2009) Meta-analysis of chemotherapy in head and neck cancer (MACH-NC): an update on 93 randomised trials and 17,346 patients. Radiother Oncol 92:4-14

3. Bourhis J, Overgaard J, Audry H et al (2006) Hyperfractionated or accelerated radiotherapy in head and neck cancer: a meta-analysis. Lancet 368:843-854

4. Beitler JJ, Zhang Q, Fu KK et al. Final results of local-regional control and late toxicity of RTOG 90-03; a randomized trial of altered fractionation radiation for locally advanced head and neck cancer. Int J Radiat Oncol Biol Phys (in press)

5. Ang KK, Harris J, Wheeler R et al (2010) Human papillomavirus and survival of patients with oropharyngeal cancer. N Engl J Med 363:24-35

6. Bourhis J, Sire C, Graff P et al (2012) Concomitant chemoradiotherapy versus acceleration of radiotherapy with or without concomitant chemotherapy in locally advanced head and neck carcinoma (GORTEC 99-02): an open-label phase 3 randomised trial. Lancet Oncol 13:145-153

7. Overgaard J, Hansen HS, Specht L et al (2003) Five compared with six fractions per week of conventional radiotherapy of squamous-cell carcinoma of head and neck: DAHANCA 6 and 7 randomised controlled trial. Lancet 362:933-940

8. Overgaard J, Mohanti BK, Begum N et al (2010) Five versus six fractions of radiotherapy per week for squamous-cell carcinoma of the head and neck (IAEA-ACC study): a randomised, multicentre trial. Lancet Oncol 11:553-560

9. Haigentz M Jr, Corry J, Strojan P, Ferlito A (2012) Easing acceleration of head and neck chemoradiotherapy. Lancet Oncol 13:113-115

10. Liang ZG, Zhu XD, Zhou ZR, Qu S, Du YQ, Jiang YM (2012) Comparison of concurrent chemoradiotherapy followed by adjuvant chemotherapy versus concurrent chemoradiotherapy alone in locoregionally advanced nasopharyngeal carcinoma: a meta-analysis of 793 patients from 5 randomized controlled trials. Asian Pac J Cancer Prev 13:5747-5752

11. Holsinger FC, Kies MS, Diaz EM Jr et al (2009) Durable longterm remission with chemotherapy alone for stage II to IV laryngeal cancer. J Clin Oncol 27:1976-1982

12. Holsinger FC, Lin HY, Bassot V, Laccourreye O (2009) Platinbased exclusive chemotherapy for selected patients with squamous cell carcinoma of the larynx and pharynx. Cancer 115:3909-3918

13. Divi V, Worden FP, Prince ME et al (2010) Chemotherapy alone for organ preservation in advanced laryngeal cancer. Head Neck 32:1040-1047

14. Forastiere AA, Shaha AR (2009) Chemotherapy alone for laryngeal preservation, is it possible? J Clin Oncol 27:1933-1934

15. Haigentz M Jr, Silver CE, Rinaldo A, Ferlito A (2010) Definitive chemotherapy: a new frontier in the fight against laryngeal cancer. Eur Arch Otorhinolaryngol 267:1-4

16. Robbins KT, Kumar P, Harris J et al (2005) Supradose intra-arterial cisplatin and concurrent radiation therapy for the treatment of stage IV head and neck squamous cell carcinoma is feasible and efficacious in a multi-institutional setting: results of Radiation Therapy Oncology Group Trial 9615. J Clin Oncol 23:1447-1454

17. Nomura K, Takahara M, Katayama A et al (2013) Intra-arterial cisplatin with concomitant radiation for advanced hypopharyngeal cancer. Laryngoscope 123:916-922

18. Kovács AF (2006) Response to intraarterial induction chemotherapy: a prognostic parameter in oral and oropharyngeal cancer. Head Neck 28:678-688
19. Regine WF, Valentino J, Arnold SM et al (2001) High-dose intraarterial cisplatin boost with hyperfractionated radiation therapy for advanced squamous cell carcinoma of the head and neck. J Clin Oncol 19:3333-3339

20. Spring PM, Valentino J, Arnold SM et al (2005) Long-term results of hyperfractionated radiation and high-dose intraarterial cisplatin for unresectable oropharyngeal carcinoma. Cancer 104:1765-1771

21. Rasch CR, Hauptmann M, Schornagel J et al (2010) Intraarterial versus intravenous chemoradiation for advanced head and neck cancer: results of a randomized phase 3 trial. Cancer 116:2159-2165

22. Ackerstaff AH, Rasch CR, Balm AJ et al (2012) Five-year quality of life results of the randomized clinical phase III (RADPLAT) trial, comparing concomitant intra-arterial versus intravenous chemoradiotherapy in locally advanced head and neck cancer. Head Neck 34:974-980

23. Janot F, de Raucourt D, Benhamou E et al (2008) Randomized trial of postoperative reirradiation combined with chemotherapy after salvage surgery compared with salvage surgery alone in head and neck carcinoma. J Clin Oncol 26:5518-5523

24. Choe KS, Haraf DJ, Solanki A et al (2011) Prior chemoradiotherapy adversely impacts outcomes of recurrent and second primary head and neck cancer treated with concurrent chemotherapy and reirradiation. Cancer 117:4671-4678

25. Strojan P, Corry J, Eisbruch A et al (2014) Recurrent and second primary squamous cell carcinoma of the head and neck: When and how to re-irradiate. Head Neck. doi:10.1002/hed.23542

26. Gugic J, Strojan P (2013) Squamous cell carcinoma of the head and neck in the elderly. Rep Pract Oncol Radiother 18:16-25

27. Bonner JA, Harari PM, Giralt J et al (2010) Radiotherapy plus cetuximab for locoregionally advanced head and neck cancer: 5-year survival data from a phase 3 randomised trial, and relation between cetuximab-induced rash and survival. Lancet Oncol 11:21-28

28. Haigentz M Jr, Cohen EE, Wolf GT, Strojan P, Eisbruch A, Ferlito A (2012) The future of induction chemotherapy for head and neck squamous cell carcinoma. Oral Oncol 48:1065-1067

29. Haddad R, O'Neill A, Rabinowits G et al (2013) Induction chemotherapy followed by concurrent chemoradiotherapy (sequential chemoradiotherapy) versus concurrent chemoradiotherapy alone in locally advanced head and neck cancer (PARADIGM): a randomised phase 3 trial. Lancet Oncol 14:257-264

30. Hitt R, Grau JJ, López-Pousa A et al (2014) A randomized phase III trial comparing induction chemotherapy followed by chemoradiotherapy versus chemoradiotherapy alone as treatment of unresectable head and neck cancer. Ann Oncol 25:216-225

31. Cohen EEW, Karrison T, Kocherginsky M et al (2012) DeCIDE: a phase III randomized trial of docetaxel (D), cisplatin (P), 5-fluorouracil (F) (TPF) induction chemotherapy (IC) in patients with $\mathrm{N} 2 / \mathrm{N} 3$ locally advanced squamous cell carcinoma of the head and neck (SCCHN). J Clin Oncol 30:5500

32. Argiris A, Li Y, Forastiere A (2004) Prognostic factors and longterm survivorship in patients with recurrent or metastatic carcinoma of the head and neck. Cancer 101:2222-2229

33. Argiris A, Ghebremichael M, Gilbert J et al (2013) Phase III randomized, placebo-controlled trial of docetaxel with or without gefitinib in recurrent or metastatic head and neck cancer: an eastern cooperative oncology group trial. J Clin Oncol 31:1405-1414

34. Shiels MS, Pfeiffer RM, Gail MH et al (2011) Cancer burden in the HIV-infected population in the United States. J Natl Cancer Inst 103:753-762

35. Haigentz M Jr (2005) Aerodigestive cancers in HIV infection. Curr Opin Oncol 17:474-478

36. McLemore MS, Haigentz M Jr, Smith RV et al (2010) Head and neck squamous cell carcinomas in HIV-positive patients: a 
preliminary investigation of viral associations. Head Neck Pathol 4:97-105

37. Haigentz M Jr, Jung G, Garg M, Owen R, Schiff B, Smith RV (2006) Squamous cell carcinoma of the head and neck (SCCHN) in patients reporting human immunodeficiency virus (HIV) infection: a retrospective case series. J Clin Oncol 24:5570

38. Klein EA, Guiou M, Farwell DG et al (2011) Primary radiation therapy for head-and-neck cancer in the setting of human immunodeficiency virus. Int J Radiat Oncol Biol Phys 79:60-64

39. Persad GC, Little RF, Grady C (2008) Including persons with HIV infection in cancer clinical trials. J Clin Oncol 26:1027-1032

40. Weiss SA, Gajavelli S, Negassa A, Sparano JA, Haigentz M (2011) Evaluation of cancer trial eligibility criteria (with focus on non-AIDS-defining cancers) for inclusion of persons with HIV infection. J Clin Oncol 29:6092

41. Ang KK, Zhang QE, Rosenthal DI et al (2011) A randomized phase III trial (RTOG 0522) of concurrent accelerated radiation plus cisplatin with or without cetuximab for stage III-IV head and neck squamous cell carcinomas (HNC). J Clin Oncol 29:5500
42. Vermorken JB, Mesia R, Rivera F et al (2008) Platinum-based chemotherapy plus cetuximab in head and neck cancer. $\mathrm{N}$ Engl $\mathrm{J}$ Med 359:1116-1127

43. Vermorken JB, Trigo J, Hitt R et al (2007) Open-label, uncontrolled, multicenter phase II study to evaluate the efficacy and toxicity of cetuximab as a single agent in patients with recurrent and/or metastatic squamous cell carcinoma of the head and neck who failed to respond to platinum-based therapy. J Clin Oncol 25:2171-2177

44. Fakhry C, Westra WH, Li S et al (2008) Improved survival of patients with human papillomavirus-positive head and neck squamous cell carcinoma in a prospective clinical trial. J Natl Cancer Inst 100:261-269

45. Haigentz M Jr, Silver CE, Corry J et al (2009) Current trends in initial management of oropharyngeal cancer: the declining use of open surgery. Eur Arch Otorhinolaryngol 266:1845-1855 